\title{
Using high frame rate CMOS sensors for three-dimensional eye tracking
}

\author{
A. H. CLARKE \\ Free University of Berlin, Berlin, Germany \\ J. DITTERICH \\ University of Munich, Munich, Germany \\ and \\ K. DRÜEN, U. SCHÖNFELD, and C. STEINEKE \\ Free University of Berlin, Berlin, Germany
}

\begin{abstract}
A novel three-dimensional eye tracker is described and its performance evaluated. In contrast to previous devices based on conventional video standards, the present eye tracker is based on programmable CMOS image sensors, interfaced directly to digital processing circuitry to permit real-time image acquisition and processing. This architecture provides a number of important advantages, including image sampling rates of up to $400 / \mathrm{sec}$ measurement, direct pixel addressing for preprocessing and acquisition, and hard-disk storage of relevant image data. The reconfigurable digital processing circuitry also facilitatesinline optimization of the front-end, time-critical processes. The primary acquisition algorithm for tracking the pupil and other eye features is designed around the generalizedHough transform. The tracker permits comprehensive measurement of eye movement (three degrees of freedom) and head movement (six degrees of freedom), and thus provides the basis for many types of vestibulo-oculomotor and visual research. The device has been qualified by the German Space Agency (DLR) and NASA for deployment on the International Space Station. It is foreseen that the device will be used together with appropriate stimulus generators as a general purpose facility for visual and vestibular experiments. Initial verification studies with an artificial eye demonstrate a measurement resolution of better than $0.1^{\circ}$ in all three components (i.e., system noise for each of the components measured as $0.006^{\circ} \mathrm{H}, 0.005^{\circ} \mathrm{V}$, and $0.016^{\circ} \mathrm{T}$. Over a range of $\pm 20^{\circ}$ eye rotation, linearity was found to be $<0.5 \%(\mathrm{H}),<0.5 \%(\mathrm{~V})$, and $<2.0 \%(\mathrm{~T})$. A comparison with the scleral search coil technique yielded near equivalent values for the system noise and the thickness of Listing's plane.
\end{abstract}

For many years, there has been a demand for accurate, noninvasive measurement of eye movement in numerous research fields. Most challenging is the requirement for accurate, three-dimensional (3-D) eye movement measurement, necessary for the study of the oculomotor and vestibular systems and for their examination in clinical diagnosis. Although there are several commercially available systems that use conventional video techniques, their standard frame rate of 50 or $60 \mathrm{~Hz}$ often proves inadequate, particularly for the correct acquisition of saccades with angular velocities of up to $500 \mathrm{deg} / \mathrm{sec}$ (Clarke, 1994). A promising alternative based on flying-spot laser scanning of the eye has recently been introduced (Irie, Wilson, Jones, Bones, \& Anderson, 2002).

This work was supported by Grant WB 0023 from the German Space Agency (Deutsches Zentrum für Luft- und Raumfahrt-DLR). The Eye Tracker Device was designed by Chronos Vision GmbH, Berlin, and qualified for deployment on the ISS by Kayser Threde GmbH, Munich, under the auspices of the DLR. A.H.C. is technical director of Chronos Vision $\mathrm{GmbH}$. Correspondence concerning this article should be addressed to A. H. Clarke, Vestibular Research Lab, Universitätsklinikum Benjamin Franklin, Freie Universität Berlin, 12200 Berlin, Germany (e-mail: clarke@medizin.fu-berlin.de).
The approach presented here provides two-dimensional (2-D) and 3-D eye position measurement at sampling rates of up to $400 / \mathrm{sec}$ and high measurement resolution $\left(<0.05^{\circ}\right.$ for all three components). The use of programmable CMOS image sensors sidesteps the inherent limitations in sampling frequency and pixel acquisition of classical video framegrabber techniques. The instrument described was developed in the first instance for integration in the Human Research Facility on the International Space Station (Clarke, 1998). To fulfill this task, many user requirements — compiled by NASA after consultation with numerous potential investigators - have been included in the design. More recently, ongoing beta-site testing at a number of vestibulo-oculomotor research labs is contributing to the refinement of the system. Besides the development of the full binocular, 3-D Eye Tracker Device (ETD), a standalone 2-D version has also evolved, typically for use as a drop-in replacement for EOG amplifier systems.

\section{Head Unit}

The head unit has been designed to permit binocular, free field-of-view recordings (Figure 1). Adjustors are provided for fitting to individual head form and for alignment 


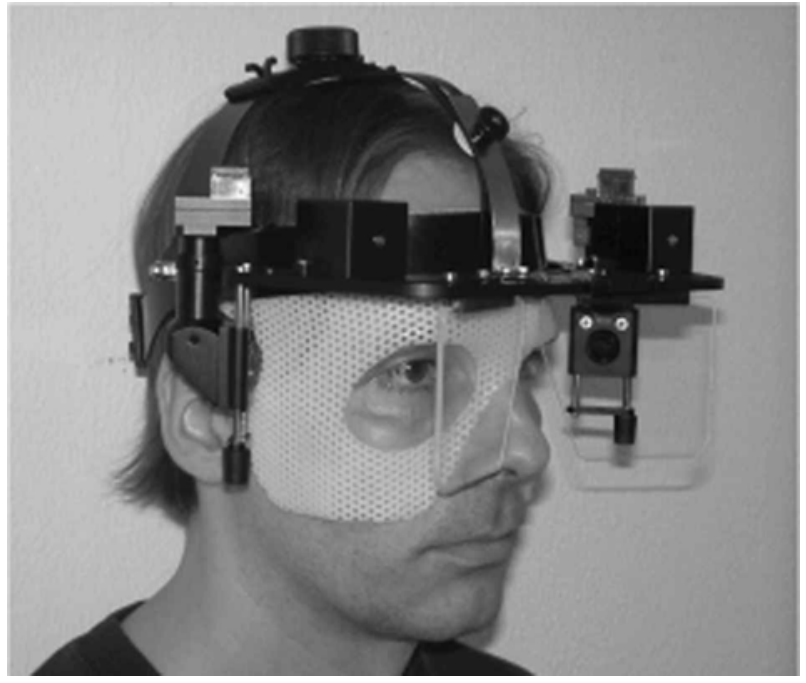

Figure 1. Eye tracker head unit with molded facemask. The cameras are mounted laterally to optimize the free field-of-view for the test subject. The image of the eye is reflected by the dichroic mirror to the optical lens and projected onto the image sensor. Triaxial angular and linear movement sensors are also mounted on the visor (cubes on top left and right of visor) to record the six degrees of freedom of head movement.

of the cameras to the right and left eyes (e.g., to encompass interocular distance, right-left asymmetries, etc.). Miniature triaxial angular rate sensors and linear accelerometers are also integrated into the head unit to permit measurement of rotation around and translation along the three orthogonal axes in space. As with all head-mounted systems, the problem of device-to-head slippage must be addressed. In the present system, the thermoplastic facemold (Figure 1) is designed both to minimize head-to-device slippage and to improve wearer comfort. In addition, provision is made for the use of biteboards and other head-stabilizing techniques (integration in flight helmet, etc.) that may be favored by the individual experimenter. Since, ultimately, no device can be perfectly fixed to the human head under normal experimental circumstances, means must be found to measure and compensate for any device slippage.

The image cameras and head movement sensors are mounted orthogonally on the visor plate to ensure a consistent frame of reference. The visor as such can be inclined to be parallel with a user-defined anatomical reference plane (e.g., Frankfurt horizontal). As is common with many video eye trackers, the eyes are illuminated by infrared-emitting diodes (set of four per eye, 940-nm wavelength). These are mounted around the camera lens. This facilitates measurements "in the dark," as is often required in vestibular and visual research and testing. In contrast to earlier infrared reflection techniques (e.g., limbus tracking), no problems arise here because of skin reflectance or radiation. The infrared image of each eye is reflected by the dichroic mirror to the optical lens and projected onto the image sensor. A near-infrared lowpass filter $(>850 \mathrm{~nm})$ is fitted in front of the camera lens to exclude any sporadic incident light. These optical elements and the cameras are arranged on the head unit to facilitate maximal field-of-view for the test subject. In practice, a field-of-view on the order of $75^{\circ}$ to the right and to the left, $25^{\circ}$ upward, and $40^{\circ}$ downward is attained.

\section{Front-End Image Processing}

Significant progress in image-processing devices is evident in the most recent CMOS image sensors. Among the most important features are the configurable acquisition of pixel-defined areas of interest and on-chip parallel processing of pixel data.

The digital cameras, which have been specially designed around state-of-the-art CMOS image sensors, include 10-bit analog-to-digital (A/D) converters and are interfaced to a dedicated processor board in the system unit via bidirectional high-speed digital transmission links (192 Mb/sec). This incorporates a field-programmable gate array (FPGA) for each image sensor and a digital signal processor (DSP) with associated storage arrays. These components are firmware programmed to perform the on-line pixel-oriented acquisition and 2-D measurement of pupil coordinates. On-line pupil detection and tracking runs automatically and requires only that the operator should align the head unit and adjust camera focus. The system architecture is illustrated in Figure 2.

Given this accurate on-line tracking facility, the region of interest (ROI) containing the pupil and the iris can be readily extracted and transferred to the host computer for display and/or storage for off-line eye position measurement. This concept eliminates the bottleneck in classical framegrabber systems that rely on the central PC processor and require frame-by-frame image acquisition.

This processing architecture facilitates image sampleand-process rates of 50/100/200/400 per second, selectable on the user interface; in addition, the binocular image sequences can be stored digitally using standard PC components, providing all the advantages of off-line evaluation. Synchronous audio recording via a standard sound card is also implemented.

\section{System Unit}

The system PC provides the graphic user interface (GUI) for display of the video eye images, data scrolls, and control elements. The Windows software also supervises on-line acquisition, data display, and storage of image data sequences. The front-end processor architecture transfers all relevant image data to the Windows datahandling process, facilitating on-line storage to hard disk for the subsequent (off-line) calculation of eye position.

From the image sequences stored on hard disk, 3-D (horizontal, vertical, and torsional) eye position is calculated using off-line algorithms. In contrast to the on-line algorithm, these permit user setting of the parameters for 2-D pupil tracking and can therefore be employed to yield higher resolution and accuracy for the calculation 


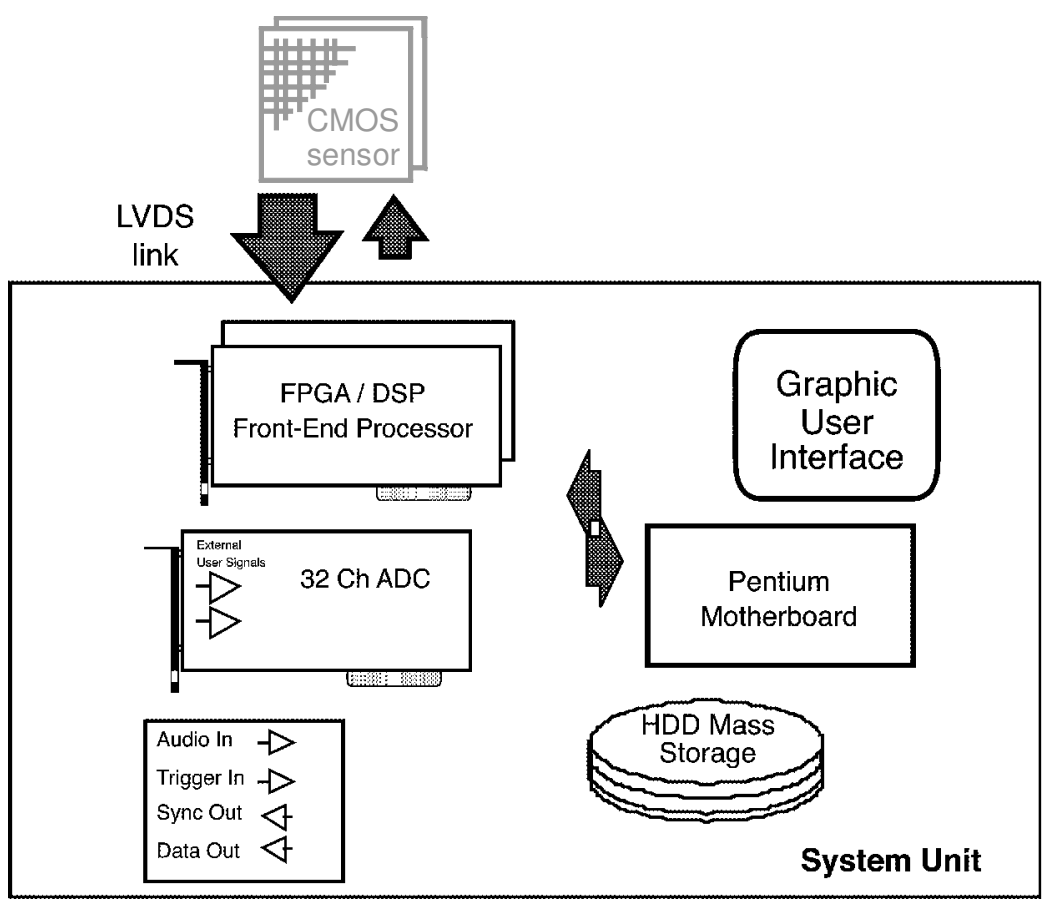

Figure 2. Outline of the principal system components of the three-dimensional Eye Tracker Device.

of horizontal and vertical coordinates (see below). This is particularly useful in cases of pupil occlusion and other artifacts. Provision is also made for setting and testing all parameters for measuring the torsional eye position.

External analog inputs are provided for the recording of signals from auxiliary equipment. For synchronization with stimulus generators and so on, the eye tracker provides sync pulses locked to the image-sampling clock. A trigger input is also provided for remote switching of hard disk recording.

\section{ACQUISITION AND MEASUREMENT SOFTWARE}

\section{On-Line Acquisition and Measurement}

The on-line acquisition and measurement software utilizes a set of front-end firmware modules for the dedicated FPGA and DSP elements. In practice, the greatest sources of error in accurate pupil detection are image artifacts caused by shadowing, reflections from tear fluid, and pupil occlusion, which result in a nonuniform distribution of pupil edge points. To overcome this, a two-pass algorithm is used in the on-line mode. During image acquisition, the relevant pixel data are transferred to the processing board. Each image is scanned for the location of the black pupil, using a template matching filter. For each scan step (1-pixel increment), a 2-D correlation is performed. The location of that point with the maximum correlation coefficient is defined in this pass as the pupil position. All matrix calculations during this pass are performed in the FPGA device.
In the region around the identified pupil center, a grid search is performed to identify potential pupil edge points and a best-fit circle is calculated with the results.

A scheduler supervises the entire on-line algorithm (i.e., transfer of camera data to the processor, initiation of the tracking algorithm, and monitoring of the processing time required for calculation of eye coordinates); this forces uniform real-time performance (i.e., output of pupil coordinates with a uniform latency time of $2 \mathrm{msec}$ for all acquisition rates).

This provides for accurate measurement of horizontal and vertical eye coordinates, which in turn, serve to define the ROI containing the pupil and the iris on a frameby-frame basis. Thus, the ROI is dynamically defined and extracted for storage on hard disk. As was described above, all time-critical data acquisition and numerical procedures are performed in the digital image cameras and the front-end processor boards. During on-line acquisition and measurement, the PC processor supervises the real-time image and data storage and the display of image inlays, data scrollers, and control elements (Figure 3).

Each data frame includes a header with time stamp, the related image data from each of the two cameras, the 2-D coordinates, and up to 32 external A/D channels. The data structure is configured according to the userselected acquisition parameters - that is, sampling rate, 2-D/3-D, and monocular/binocular acquisition. In addition, the 2-D eye position coordinates and all A/D data are stored in ASCII table format. 


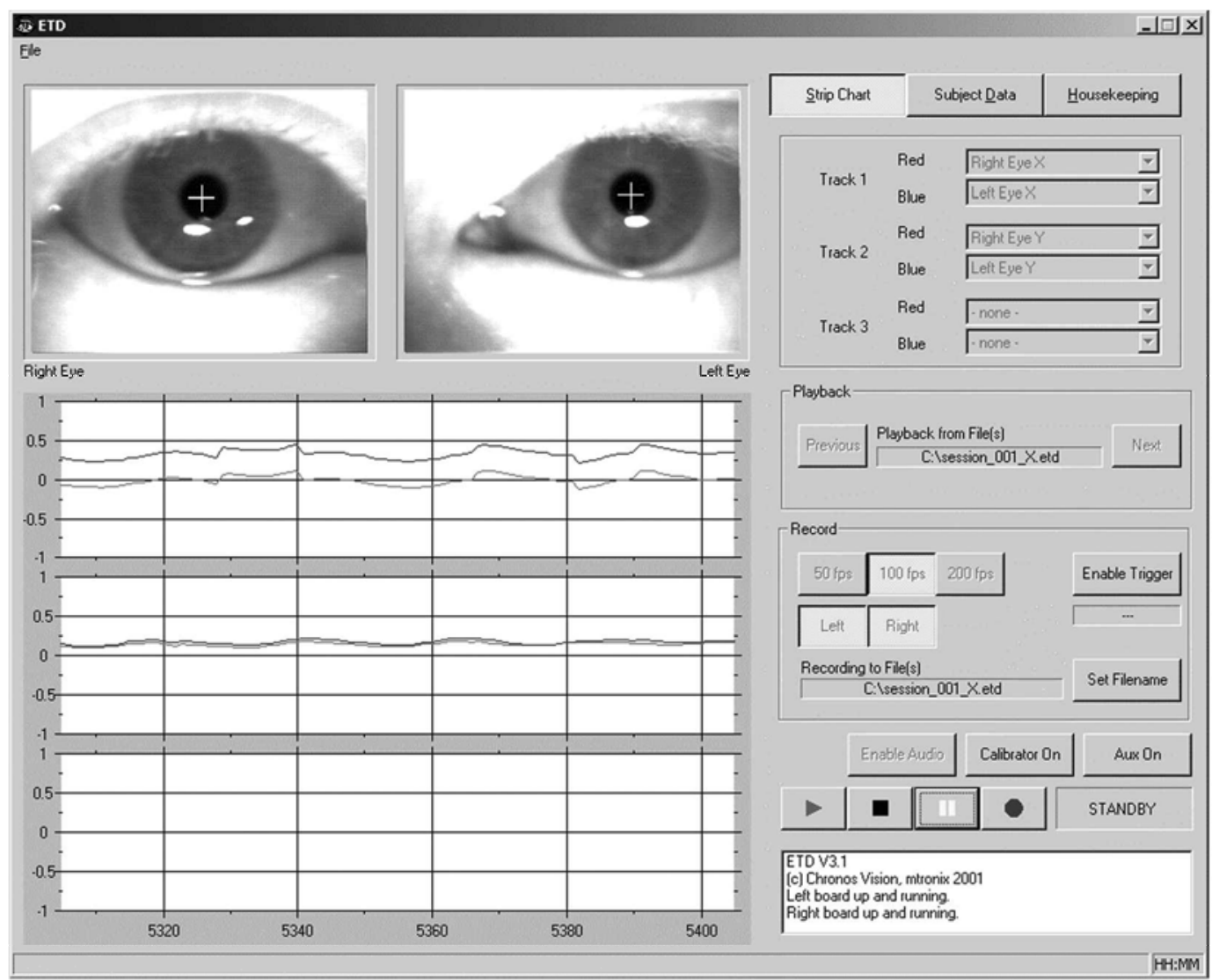

Figure 3. Screen shot of the graphic user interface (GUI) for on-line measurement and monitoring of eye movements and measured coordinates (superimposed as crosshairs on the video inlays and displayed on data scrollers). This GUI also provides for selection of acquisition parameters (sampling rate, mono/binocular, audio).

\section{Off-Line Image Evaluation}

In many applications, it is expedient to video-record the eye movement behavior of interest, as opposed to making a one-off measurement of eye position. On the one hand, a thorough off-line evaluation can be performed and, if necessary, repeated for verification, and on the other hand, critical experiment time is not expended with setting up on-line evaluation parameters. This is particularly true in the clinic and in the spaceflight situation, in which astronaut time is always at a premium. Furthermore, the digital recording facility enables the user to archive image sequences of interest for subsequent use-for example, for considerably more convincing demonstration or teaching purposes.

For the off-line evaluation of the 3-D eye position, the user can choose between the stored 2-D coordinates, as measured with the on-line tracking algorithm, and the calculation of horizontal and vertical eye position with one of the two off-line algorithms.

Convention for describing 3-D eye position. The angular orientation of the eye can be specified by a Cartesian coordinate system that is fixed in the eye, with respect to a second set of space-fixed coordinates, where both coordinate frames have a common origin. Since the eyeball is limited to three degrees of rotational freedom, only three independent parameters are required to specify the orientation of the eye in 3-D space. The most common description used in oculomotor research involves Euler angles. This parameterization can be derived from the $3 \times 3$ direction cosine matrix, which in turn, is derived from the projections of unit normal vectors aligned with the three eye-fixed axes onto the head-fixed coordinate frame. In the following, the eye is assumed to be a perfect sphere exhibiting ideal ball-and-socket behavior. All eye movements can therefore be described as pure rotations around the center of this sphere (for details, see Haslwanter, 1995).

Off-line pupil tracking. The majority of algorithms for calculation of eye position are based on determination of the center of the pupil. Although the simpler approaches (e.g., pupil centroid) can be rejected for their artifact susceptibility, a number of more robust and more accurate circle approximation techniques for the pupil perimeter have been developed (e.g., Barbur, Thomson, \& Forsyth, 1987; Groen, Bos, Nacken, \& de Graaf, 1996; Sung \& Reschke, 1997; Zhu, Moore, \& Raphan, 1999). 


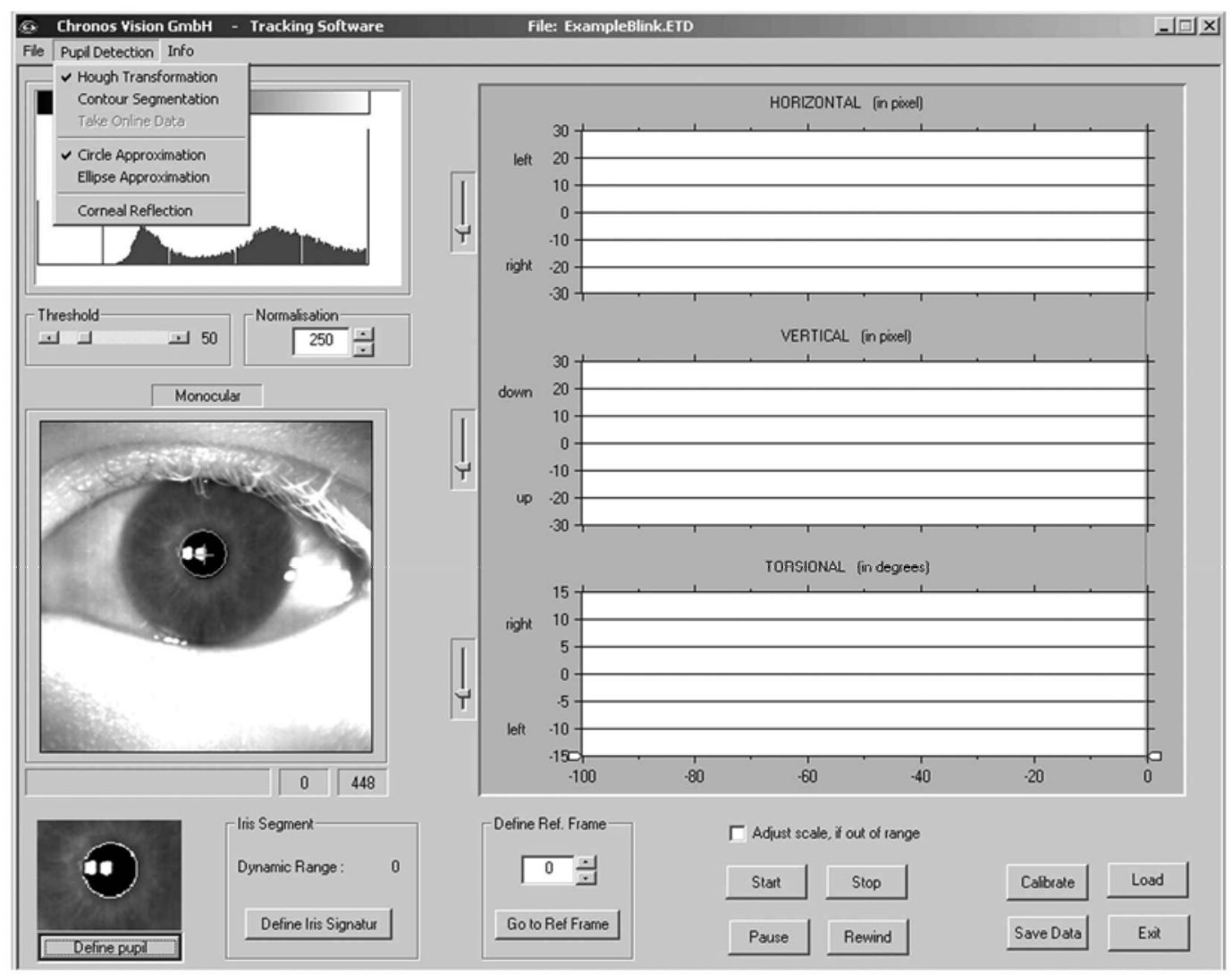

Figure 4. Screen shot of the graphic user interface of the off-line software, with user control elements, interactive windows, and three-dimensional data scrollers.

It has further been demonstrated that ellipse fitting (e.g., Pilu, Fitzgibbon, \& Fisher, 1996) represents a better approximation to the pupil form of most eyes and allows for compensation of any geometric distortion during eye rotation (Moore, Haslwanter, Curthoys, \& Smith, 1996).

Besides the problem of pupil form, image artifacts must be overcome. The first off-line algorithm is based on the Hough transform technique. It permits the user to adjust image contrast, edge detection threshold, and circle/ ellipse detection. The second off-line pupil-tracking algorithm is based on contour segmentation. Thus, the image is scanned for transitions across a predefined gray-level threshold and connects contiguous strings of transition points. Those strings with uniform curvature are assumed to belong to the pupil perimeter. A number of heuristic matching criteria are checked to obtain the best-fitted circle or ellipse.

In contrast to the on-line algorithm, which employs automatic parameter setting, the off-line software permits comprehensive interactive adjustment of the pupil segmentation parameters via the user interface (Figure 4).

Calibration for eye position calculation. The offline software includes a calibration routine for estimating the eye model parameters from a standard fixation pattern (typically, $\pm 10^{\circ} \mathrm{right} / \mathrm{left}$ and up/down). The es- timated parameters are stored in a configuration file and are used for correction of the geometric projection error in tertiary eye positions and for the transformation from pixel to Euler coordinates. The user interface for generating the eye model is illustrated in Figure 5.

Calculating 3-D eye position. Two approaches have been implemented for evaluation of 3-D eye position, one based on iris segment correlation and the other using scleral marker tracking.

Iris segment correlation. This is an implementation of the polar correlation algorithm first described by Hatamian and Anderson (1983). The approach exploits the fact that the relevant information for determining ocular torsion is contained in the natural landmarks of the iris. In terms of polar coordinates, the premise is that this information is reflected by the angular, rather than the radial, component of the iris image. Thus, in addition to accurate determination of the center of the pupil, this concept requires the extraction of a natural luminance profile derived from circular sampling around the iris.

By deriving from the center of the pupil for the current image, the coordinates of a circular sample in the iris are calculated.

During an initial set-up procedure, the radius and extent of the annulus are selected interactively by the op- 


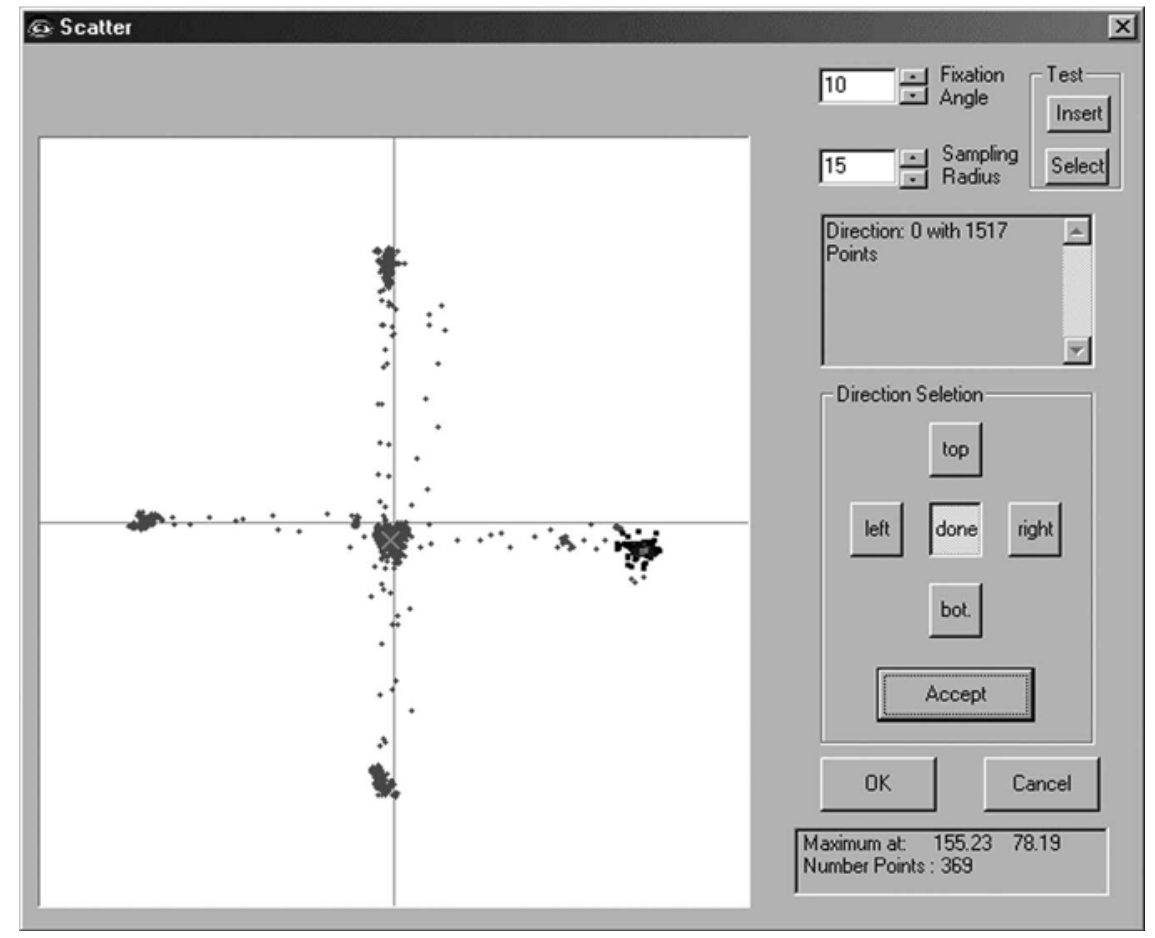

Figure 5. Calibration window in off-line software. The scattergram is generated from the recorded calibration sequence. Each of the position clusters is allocated by user interaction to one of the predefined fixation points (typically, as is shown, $\pm 10^{\circ} \mathrm{left} / \mathrm{right}$ and up/down).

erator with the eye in reference position (straight and level) - that is, presumably with zero torsion, as is illustrated in Figure 6.

The iris luminance profile, or signature, from the reference frame is employed as zero reference for the subsequent frame-by-frame correlation. During the frameby-frame evaluation, the corresponding subset of pixels is addressed, and their luminance value is extracted. Calculation of ocular torsional position is then performed by one-dimensional cross-correlation of the current iris signature against the predetermined reference signature. The delay to the peak correlation value corresponds directly to the torsional displacement. During the frame-by-frame evaluation, the nonlinear distortion of the iris segment (owing to the projection of the spherical eyeball onto the image plane) is corrected on the basis of the calibration model (Moore et al., 1996; Peterka \& Merfeld, 1996).

The user interface is designed so that a number of approaches can be realized with appropriate postprocessing. For example, the more complex technique described by Bos and de Graaf (1994) can be performed. They have shown that when the pupil center is not well defined, selection of a short iris profile can lead to large errors. As a solution, Groen et al. (1996) demonstrated that an iterative weighted sinusoidal fit of torsion estimates about the full $360^{\circ}$ yields a more accurate measure of torsion. This technique is useful in cases of pupil occlusion because of droopy eyelids. These authors also argue that for the elimination of illumination artifacts, the crosscorrelation technique should be applied on multiple iris segments distributed over a large tangential area of the iris.

Ultimately, the results are highly dependent on the magnitude of the gray-level contrast signal selected from the natural iris. This usually amounts to between 20 and 35 gray-level increments but can be less in eyes with low contrast under infrared illumination. The scleral marking technique described in the next section offers a practical workaround in such cases. Extracting a profile through the marker on the sclera, rather than from within the iris, yields a clearly defined landmark and a high-contrast signal (typically, $>40$ gray-level increments), so that the signal-to-noise ratio with the polar correlation algorithm is greatly enhanced.

Scleral marker tracking. The scleral markers are applied directly to the sclera close to the limbus, using a sterile surgical marker pen. This is usually performed after anaesthetizing the surface of the eye. Care must be taken not to contact the sensitive cornea.

As a precursor to this technique, Young et al. (1981) described the use of landmarked contact lenses. An improvement was actually employed in the present search coil comparison, in which the annuli were prepared with infrared markers.

The present algorithm, which requires the application of such scleral markers or prepared contact rings, exploits the fact that given at least two fixed landmarks on 


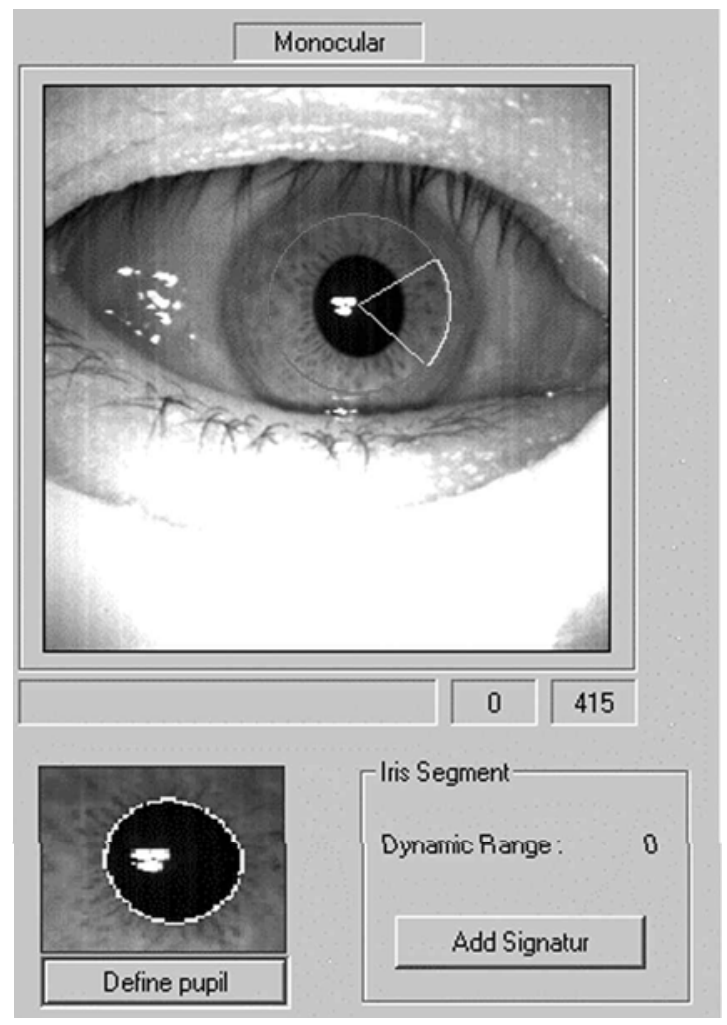

Figure 6. Illustration of iris segment selection (performed interactively). The selected segment is then used for polar correlation, to yield the torsional eye coordinate.

the eyeball, its 3-D position can be calculated. This approach was first proposed by Nakayama (1974) and was reexamined by Ott, Gehle, \& Eckmiller (1990). In practice, the approach requires accurate determination of the pupil coordinates and those of at least two scleral markers.

During initial setup, two scleral markers and the pupil are manually segmented from a reference image, and local luminance thresholds are set for each of them, as is illustrated in Figure 7. On the basis of the coordinates of the markers in relation to pupil position, local ROIs are defined. Throughout the sequence, the scleral markers are localized within their respective ROIs and are segmented by local thresholding. During evaluation, the horizontal and vertical coordinates of the pupil are displayed together with the angle of the connecting line between the scleral markers.

\section{VERIFICATION OF SYSTEM PERFORMANCE}

\section{Measurements With Artificial Eye Model}

In order to test objectively the performance of the ETD acquisition and evaluation components, it is necessary to generate predefined eye movements. In contrast to test sequences recorded from human eyes, where, ultimately, the true position of the eye remains uncertain, the artifi- cial eye approach yields sequences of well-defined eye position.

For this purpose, a model eye mounted in a three-axis gimbal and aligned orthogonally to the imaging camera was employed (Figure 8 ). This permits calibrated positioning around each of the three axes in high-resolution increments (i.e., $34 \times 10^{-6^{\circ}}$ ). For these tests, the artificial eye (25-mm diameter; iris diameter, $12 \mathrm{~mm}$; pupil diameter, $7 \mathrm{~mm}$ ) was positioned at the same distance from the camera as with the trackers head unit. Clear iral landmarks were prepared on the artificial eye, which yielded a graylevel contrast of at least 35 increments. Throughout the artificial eye study, the off-line Hough transform technique was used for evaluation of pupil coordinates, and the polar correlation technique was used for the torsional coordinate.

Measurement resolution. This is a function of both pixel resolution of the sensor chip and the algorithms employed. In Figures 9A, 9B, and 9C, the measurement resolution for the horizontal, vertical, and torsional position is demonstrated to be considerably better than $0.1^{\circ}$. The system noise for each of the components, expressed as standard deviation about the mean, amounts to $0.006^{\circ}(\mathrm{H}), 0.005^{\circ}(\mathrm{V})$, and $0.016^{\circ}(\mathrm{T})$.

Accuracy and linearity. The required accuracy for the NASA application was specified as $<5 \%$ for the range of $\pm 10^{\circ}$ and as $10 \%$ beyond that range. To obtain a measure of the accuracy and linearity of the ETD, eye positions were measured over the range of $\pm 40^{\circ}$ in $2^{\circ}$ steps around the horizontal and vertical axes and $\pm 20^{\circ}$ for the torsional axis. The results are presented in Figure 10 and are summarized in Table 1.

\section{Comparison With Scleral Search Coils ${ }^{1}$}

The scleral search coil (SSC) technique is generally accepted as the gold standard for measurement of the 3-D position of the eye. In order to evaluate the performance of the ETD, it was therefore expedient to compare its performance with that of the SSC technique. In the study, the accuracy of measured eye position during fixation of predefined targets was examined. Subjects were required to fixate a series of points arranged in $5^{\circ}$ intervals over a $\pm 20^{\circ}$ horizontal $\times \pm 15^{\circ}$ vertical matrix. Each subject performed four scans of the point matrix: starting top left and scanning left to right, starting bottom right and scanning upward, starting bottom right and scanning right to left, and starting top left and scanning downward. Prior to the matrix trials, calibration routines were carried out for the coils and the eye tracker. The tar-

Table 1

Measurement Accuracy of the Eye Tracker Device Expressed as Measurement Error in Percentage of Measurement Range

\begin{tabular}{cccc}
\hline $\begin{array}{c}\text { Measurement } \\
\text { Range }\end{array}$ & Horizontal & Vertical & Torsional \\
\hline $\pm 10^{\circ}$ & $<1.0$ & $<1.0$ & $<1.5$ \\
$\pm 20^{\circ}$ & $<0.5$ & $<0.5$ & $<2.0$ \\
$\pm 30^{\circ}$ & $<0.4$ & $<0.4$ & - \\
$\pm 40^{\circ}$ & $<1.0$ & $<1.25$ & - \\
\hline
\end{tabular}




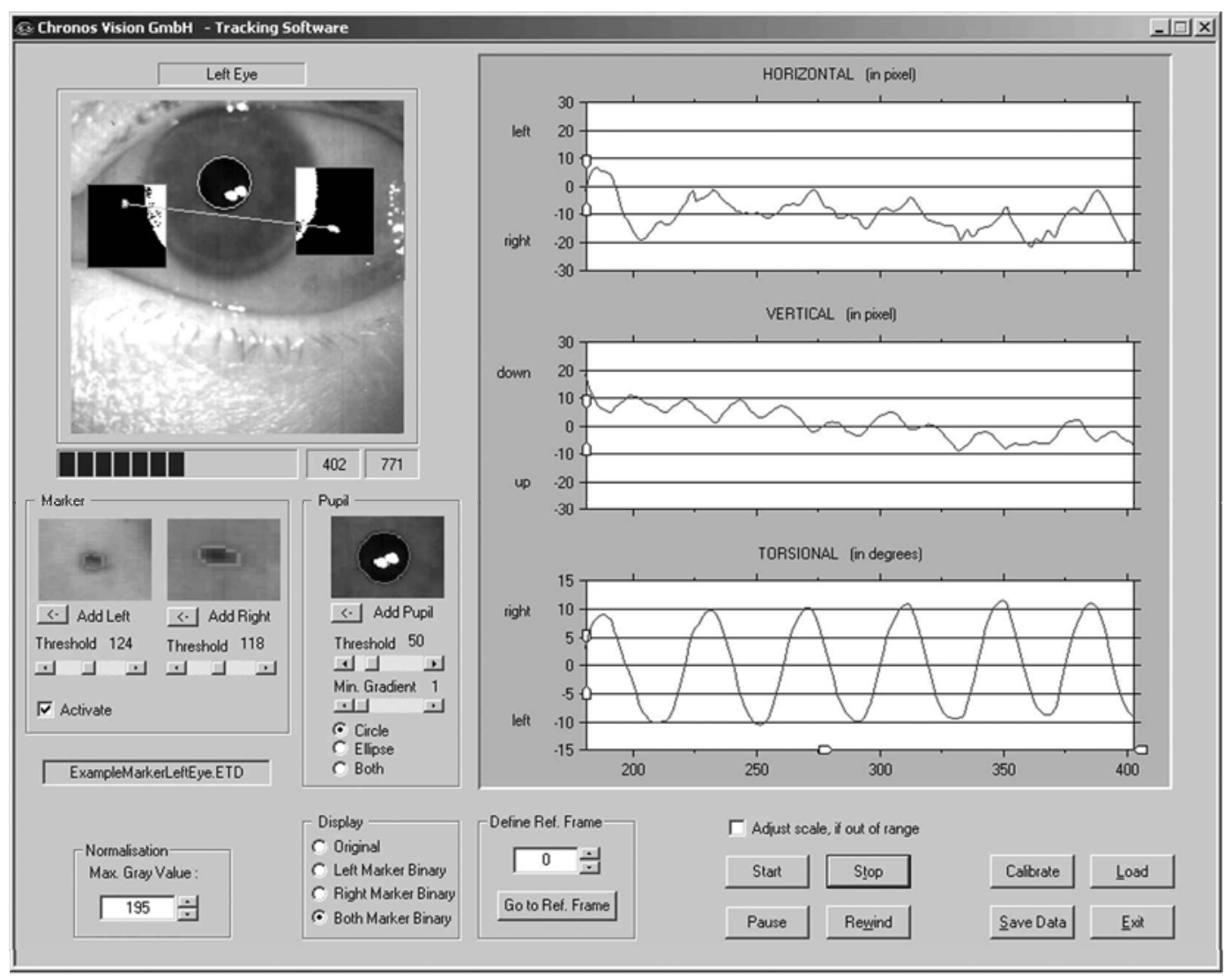

Figure 7. Scleral marker tracking, illustrating pupil and marker tracking and the resultant three-dimensional eye position traces. In the video inlay, the two regions of interest enclosing the markers are highlighted; the thresholded markers are displayed on the video inlay.

get points were generated using a laser beam guided by a dual-axis galvanometer and projected to a surface at $1.38 \mathrm{~m}$ in front of the subject. Measurement data were obtained from 6 healthy test subjects. The data presented here were all obtained from simultaneous measurements on the same eye with the ETD and the SSC system. This approach is similar to that reported recently by van der Geest and Frens (2002), who compared the performance of a 2-D video-based eye tracker with that of search coils.

The SSC data were obtained using a three-field system (Remmel Labs) and 3-D search coils specially prepared with embedded black markers (Skalar Medical BV). The six analog signals were sampled at $1 \mathrm{kHz}$ and digitized with 12-bit resolution. Image sequences of the same eye were recorded simultaneously with the ETD system at a sampling rate of $50 \mathrm{~Hz}$. Eye position was calculated offline by tracking the markers embedded in the coil. This ensured that the ETD actually measured the movements of the coil.

3-D accuracy. According to Listing's law, the eye assumes a defined torsional rotation for each 2-D position. By using quaternions to describe the 3-D eye position, the torsional position is approximately a linear function of the horizontal and vertical coordinates. This relationship provides an objective basis for the evaluation of the 3-D accuracy.

For the ETD and SSC data, Listing's plane was calculated, using the three components of the quaternions $Q_{\mathrm{H}}$, $Q_{\mathrm{V}}$, and $Q_{\mathrm{T}}$. The three plane parameters $p_{1}, p_{2}$, and $p_{3}$ were determined by a least squares fit:

$$
\begin{gathered}
\stackrel{\circ}{\mathrm{T}}=p_{1} \cdot Q_{\mathrm{H}}+p_{2} \cdot Q_{\mathrm{V}}+p_{3} \\
\sum\left(Q_{\mathrm{T}}-\stackrel{\circ}{\mathrm{T}}^{2}\right)^{2}=\min ,
\end{gathered}
$$

with $\stackrel{\circ}{\mathrm{T}}_{\mathrm{T}}$ being the expected torsional component of the quaternion based on Listing's law.

The thickness of Listing's plane was defined as

$$
\sqrt{\frac{\sum\left(Q_{\mathrm{T}}-\stackrel{\circ}{Q}_{\mathrm{T}}\right)^{2}}{n}},
$$

where $n$ is the total number of measured eye positions, and was taken as a measure of the deviation from Listing's law. These deviations can result from both physiological factors and inaccuracies of the measurement system. Recent studies have demonstrated that, under strict experimental conditions, the thickness of Listing's plane, when measured 


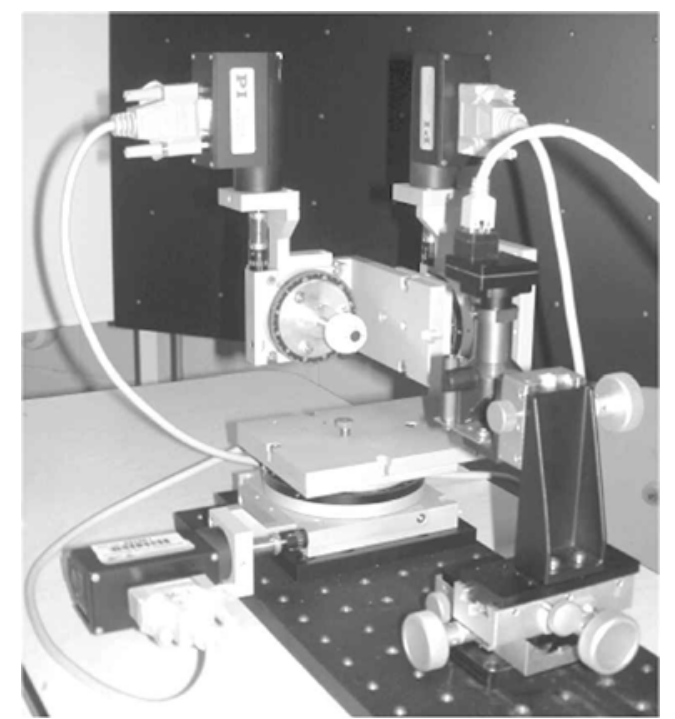

Figure 8. Illustration of motorized three-dimensional gimbal (Physik Instrumente $\mathbf{G m b H}$ ), for precision positioning of the artificial eye and ETD camera.

using the search coil technique, is typically between $1.5^{\circ}$ (Tweed \& Vilis, 1990 ) and $0.7^{\circ}-0.9^{\circ}$ (Desouza, Nicolle, \& Vilis, 1997; Minken, Van Opstal, \& van Gisbergen, 1993; Straumann, Zee, Solomon, Lasker, \& Roberts, 1995).

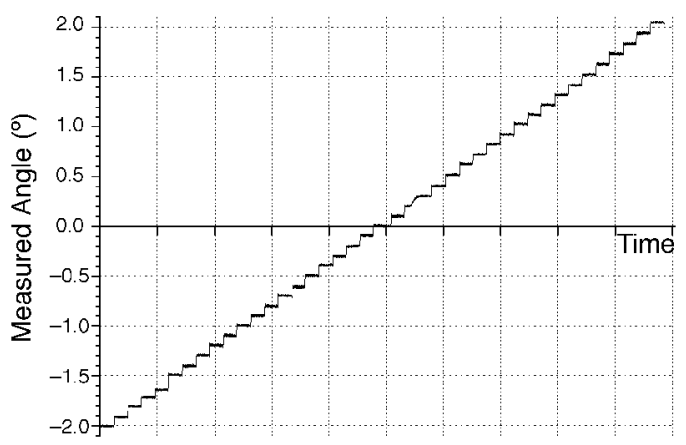

A: Horizontal Rotation

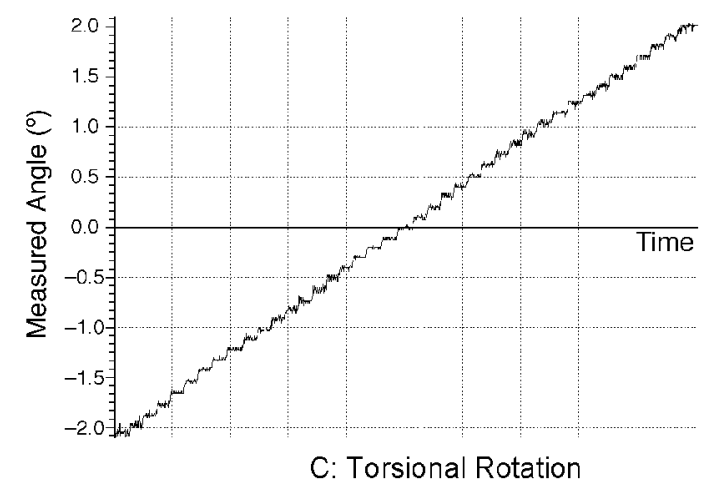

It should be noted that in the present comparative study, it was considered critically important that recordings were made simultaneously with both systems.

Over the group of 6 test subjects, the mean value for the thickness of Listing's plane amounted to $1.32^{\circ}(\mathrm{min}$, $0.84^{\circ} ; \max , 1.82^{\circ}$ ) for the SSC system and $1.34^{\circ}$ (min, $0.47^{\circ} ;$ max, $1.93^{\circ}$ ) for the ETD. The relatively large variability between subjects can be attributed to small spontaneous head movements during the measurement, since a chinrest, rather than a biteboard, was used. However, of primary importance is the finding that no statistically significant difference was found between the two techniques ( $p=.92$, using the Wilcoxon matched-pair test).

System noise. System noise was evaluated by calculating the standard deviation of eye position over each fixation interval. It should be noted that this measure is dependent on both the system noise of the measurement system and the stability of each individual's fixation. However, since simultaneous recordings on the same eye were made, the relevant measure is the difference between the two systems, rather than the magnitude.

The inherent system noise for the SSC technique has been estimated to be on the order of $0.004^{\circ}$ (Collewijn, van den Mark, \& Jansen, 1975). The measurements with the artificial eye yield a realistic estimate of the inherent system noise of the eye tracker. Drawing from the results shown in Figure 10 for the artificial eye measurements, the inherent system noise with a stationary artificial eye

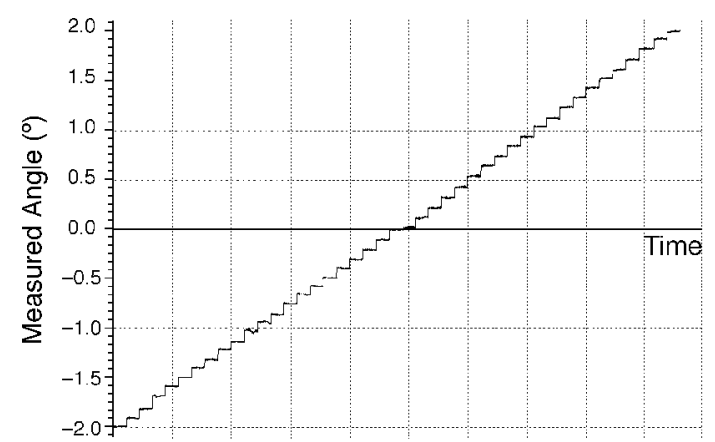

B: Vertical Rotation

Figure 9. Measurement resolution: The data show the measured angle as the artificial eye is stepped through the range from $-2.0^{\circ}$ to $+2.0^{\circ}$ in $0.1^{\circ}$ increm ents. The panels show (A) the horizontal, $(B)$ the vertical, and (C) the torsional component of eye movement. The system noise for each of the components amounts to $0.006^{\circ}\left(\mathrm{H}^{\circ}\right), \mathrm{0.005}^{\circ}$ (V), and $\mathrm{O.016}^{\circ}(\mathrm{T})$. 

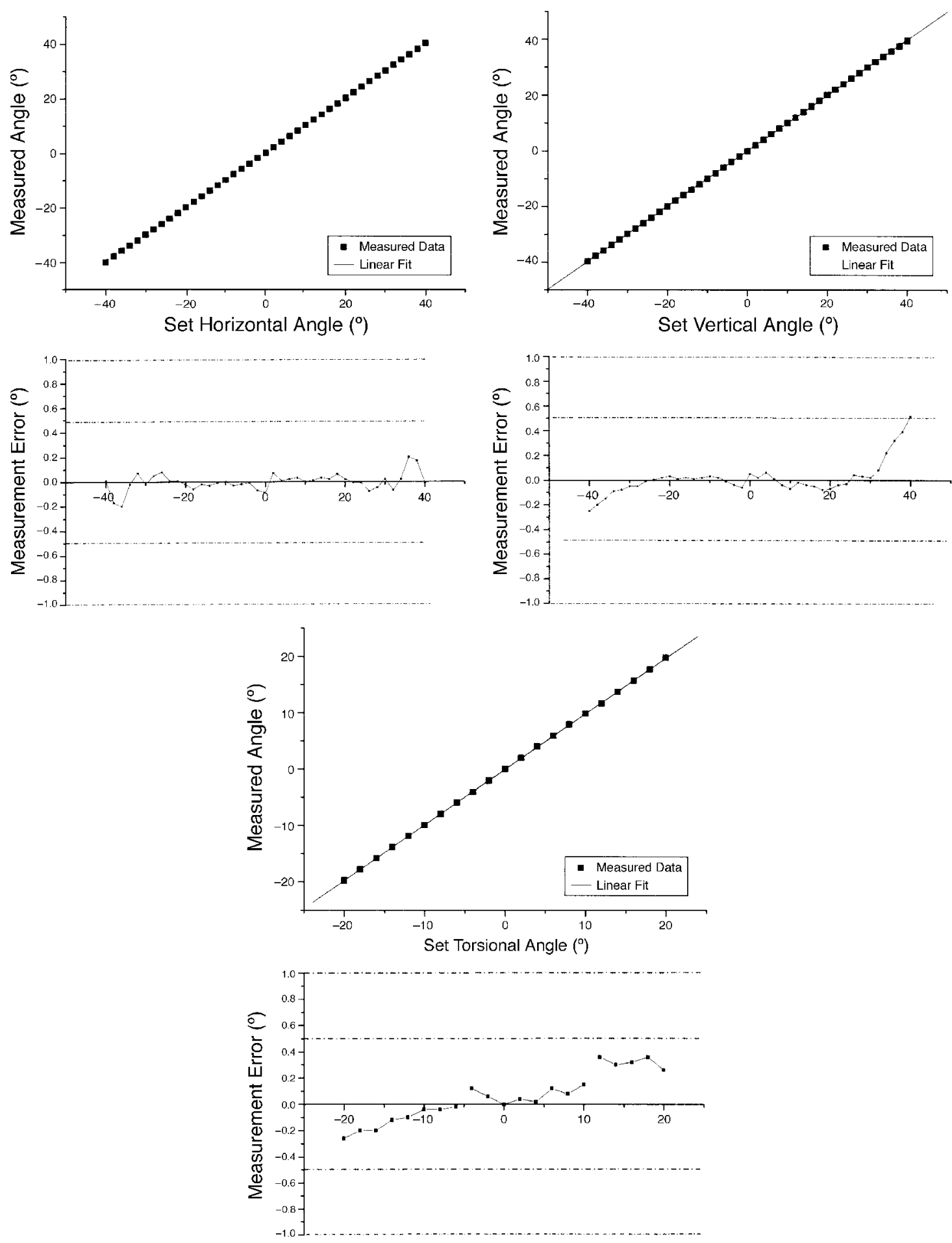

Figure 10. The upper panels show the average measured angle against the set angle for the horizontal, vertical, and torsional components. The lower panels illustrate the accuracy for each component, expressed as measurement error.

amounts to $0.006^{\circ}(\mathrm{H}), 0.005^{\circ}(\mathrm{V})$, and $0.016^{\circ}(\mathrm{T})$. The results, as summarized in Table 2 , yield an effective system noise on the order of $0.1^{\circ}$. This indicates that the inherent system noise of both systems is masked by phys- iological noise, or natural fluctuations in eye position. This indicates that for practical purposes, the ETD and the SSC techniques provide near-identical performance for 3-D eye position measurement. 
Table 2

Median and Minimum/Maximum Values for the System Noise Measured for Each Eye Position Component During Simultaneous Measurements with Scleral Search Coil and the Eye Tracker Device

\begin{tabular}{lccccccc}
\hline & \multicolumn{3}{c}{ SSC Noise $\left(^{\circ}\right)$} & & \multicolumn{3}{c}{ Tracker Noise $\left(^{\circ}\right)$} \\
\cline { 2 - 4 } \cline { 7 - 8 } Component & Median & Min & Max & & Median & Min & Max \\
\hline Horizontal & 0.08 & 0.05 & 0.18 & & 0.11 & 0.08 & 0.21 \\
Vertical & 0.08 & 0.06 & 0.10 & & 0.09 & 0.06 & 0.10 \\
Torsional & 0.09 & 0.07 & 0.10 & & 0.07 & 0.06 & 0.08 \\
\hline
\end{tabular}

Note-Measurements were performed on 6 human subjects. The individual values were calculated as the standard deviation around the mean during each fixation interval.

\section{CONCLUDING REMARKS}

The state-of-the-art 3-D eye tracker presented here utilizes CMOS imaging technology to provide an accurate, high frame rate, 3-D measurement of eye position. This represents a new generation in image-based eyetracking equipment, combining all of the features of previous video-oculography systems with the advantages of digital cameras and digital storage of image sequences.

As a multiple-purpose eye movement monitoring device, the system provides the user with on-line measurement of 2-D eye position, binocular digital image recording, and off-line evaluation of 3-D binocular eye position. These features permit reviewing of the eye image sequences and repeated evaluation, if desired, with different algorithms. Furthermore, the straightforward setup procedure and the noninvasive nature of the device are particularly attractive for use in the clinic, where due consideration must be given to patient comfort, and in many other experimental situations in which operating conditions preclude the more invasive SSC technique.

As reflected by the artificial eye measurements and the SSC comparison with human eyes, the tracker performs accurately over the physiological range of natural eye movements.

\section{REFERENCES}

Ballard, D. H., \& Brown, C. M. (1982). Computervision. Englewood Cliffs, NJ: Prentice-Hall.

Barbur, J., Thomson, W. D., \& Forsyth, P. M. (1987). A new system for the simultaneous measurement of pupil size and two-dimensional eye movements. Clinical Vision Sciences, 2, 131-145.

Bos, J. E, \& DE GrAAF, B. (1994). Ocular torsion quantification with video images. IEEE Transactions on Biomedical Engineering, 41, 351-357.

Clarke, A. H. (1994). Image processing techniques for the measurement of eye movement. In J. Ygge \& G. Lennerstrand (Eds.), Eye movements in reading (pp. 21-38). Oxford: Pergamon.

Clarke, A. H. (1998). Vestibulo-oculomotor research and measurement technology for the space station era. Brain Research Reviews, 28, 173-184.

Collewijn, H., van den Mark, F., \& Jansen, T. C. (1975). Precise recording of human eye movements. Vision Research, 15, 447-450.
Desouza, J. F. X., Nicolle, D. A., \& Vilis, T. (1997). Task-dependent changes in the shape and thickness of Listing's plane. Vision Research, 37, 2271-2282.

Duda, R. O., \& Hart, P. E. (1973). Pattern classification and scene analysis. New York: Wiley.

Groen, E., Bos, J. E., Nacken, P. F. M., \& de Graaf, B. (1996). Determination of ocular torsion by means of automatic pattern recognition. IEEE Transactions on Biomedical Engineering, 43, 471-479.

HASLWANTER, T. (1995). Mathematics of three-dimensional eye rotations. Vision Research, 35, 1727-1739.

Hatamian, M., \& ANDERson, D. J. (1983). Design considerations for a realtime ocular counterroll instrument. IEEE Transactions on Biomedical Engineering, BME-13(2), 65-70.

Hough, P. V. C. (1962). Methods and means for recognising complex patterns. US Patent 3069654.

Irie, K., Wilson, B. A., Jones, R. D., Bones, P. J., \& Anderson, T. J. (2002). A laser-based eye-tracking system. Behavior Research Methods, Instruments, \& Computers, 34, 561-572.

Minken, A. W. H., Van Opstal, A. J., \& Van Gisbergen, J. A. M. (1993). Three-dimensional analysis of strongly curved saccades elicited by double-step stimuli. Experimental Brain Research, 93, 521-533.

Moore, S. T., Haslwanter, T., Curthoys, I. S., \& Smith, S. T. (1996). A geometric basis for measurement of three dimensional eye position using image processing. Vision Research, 36, 445-459.

NAKAYAma, K. (1974). Photographic determination of the rotational state of the eye using matrices. American Journal of Optometry \& Physiological Optics, 51, 736-741.

Ott, D., Gehle, F., \& ECKMILleR, R. (1990). Video-oculographic measurement of 3-dimensional eye rotations. Journal of Neuroscience Methods, 35, 229-234.

Parker, P. J. R. (1994). Practical computer vision using C. New York: Wiley.

Peterka, R. J., \& Merfeld, D. M. (1996). Calibration techniques for video-oculography. Journal of Vestibular Research, $\mathbf{6}, \mathrm{S} 75$.

Pilu, M., FitzGibbon, A., \& Fisher, R. (1996). Ellipse-specific direct least-square fitting. Paper presented at the IEEE International Conference on Image Processing, Lausanne.

Straumann, D., Zee, D. S., Solomon, D., Lasker, A. G., \& Roberts, D. C. (1995). Transient torsion during and after saccades. Vision Research, 35, 3321-3334.

Sung, K., \& ReschKe, M. (1997). A model-based approach for the measurement of eye movements using image processing (NASA Tech. Paper 3680). Washington, DC: U.S. Government Printing Office.

TweED, D., \& Vilis, T. (1990). Geometric relations of eye position and velocity vectors during saccades. Vision Research, 30, 111-127.

vAN DER GeEST, J. N., \& Frens, M. A (2002). Recording eye movements with video-oculography and scleral search coils: A direct comparison of two methods. Journal of Neuroscience Methods, 114, 185-195. Young, L. R., Lichtenberg, B. K., Arrott, A. P., Crites, T. A., OMAN, C. M., \& EDELMAN, E. R. (1981). Ocular torsion on Earth and in weightlessness. In B. Cohen (Ed.), Vestibular and oculomotor physiology: International meeting of the Barany Society (Annals of the New York Academy of Sciences, Vol. 374, pp. 80-92). New York: New York Academy of Sciences.

Zhu, D., Moore, S. T., \& Raphan, T. (1999). Real-time torsional eye position calculation from video images. Society for Neuroscience $A b$ stracts, 25, 1650.

\section{NOTE}

1. These measurements were carried out independently at the Center for Sensorimotor Research, Neurology Department of the University of Munich. 


\section{APPENDIX \\ The Hough Transform Technique}

The Hough transform (Hough, 1962) is described in detail in many textbooks on computer vision (e.g., Ballard \& Brown, 1982; Parker, 1994). There are also a number of excellent Web sites describing its implementation (e.g., www.dai.ed.ac.uk). It is a possibilistic method for locating features of arbitrary shape in an image. In practice, it is often used for finding straight lines, circles, or ellipses. For the present application, its inherent robustness is particularly useful when pupil occlusion occurs. The technique is outlined here for circle detection, as first described by Duda and Hart (1973).

A circle can be represented as

$$
(x-a)^{2}+(y-b)^{2}-r^{2}=0
$$

or, in polar coordinates, as

$$
\begin{aligned}
& x=a+r \cos (q) \\
& y=b+r \sin (q) .
\end{aligned}
$$

This model has three parameters: two parameters for the center of the circle and one parameter for the radius of the circle. The direction of the vector from the center of the circle to each edge point is determined by the gradient angle, leaving the value of the radius as the only unknown parameter.

The intensity gradient is calculated across the image and then thresholded to yield the locations of potential edge points. The positions of possible centers-namely, those points at a distance $r$ from each detected edge point-are accumulated in a parameter space. Here, $r$ is the anticipated circle radius. The parameter space is usually defined to be congruent to the image space. In a final step, the accumulated parameter space is searched for peaks that correspond to the center(s) of the circular object(s), and the "candidate" with the highest frequency of votes is selected. A number of refinements have been proposed to reduce the influence of edge point noise (e.g., Sobel filtering) and to reduce the number of operations in the accumulation of large numbers of points (or votes) in the parameter space.

(Manuscript received March 20, 2002; revision accepted for publication August 29, 2002.) 